\title{
Physician Support of Smoking Cessation After Diagnosis of Lung, Bladder, or Upper Aerodigestive Tract Cancer
}

\author{
Amanda Farley, $P b D^{1}$ \\ Constantinos Koshiaris, MSc ${ }^{2}$ \\ Jason Oke, $P b D^{2}$ \\ Ronan Ryan, $\mathrm{PbD}^{1}$ \\ Lisa Szatkowski, $\mathrm{PbD}^{3}$ \\ Richard Stevens, $\mathrm{PbD}^{2}$ \\ Paul Aveyard, FRCGP2 \\ 'Institute of Applied Health Research, \\ University of Birmingham, Edgbaston, \\ Birmingham, United Kingdom \\ ${ }^{2}$ Nuffield Department of Primary Care \\ Health Sciences, UK Centre for Tobacco \\ and Alcohol Studies, University of Oxford, \\ Oxford, United Kingdom \\ ${ }^{3}$ Division of Epidemiology and Public \\ Health, UK Centre for Tobacco and \\ Alcohol Studies, University of Nottingham, \\ Nottingham, United Kingdom
}

Conflicts of interest: P.A. reports grants from the Medical Research Council (MRC), National Institute for Health Research (NIHR), Cancer Research UK (CRUK), Economic and Social Research Council, and UK Centre for Tobacco and Alcobol Studies during the conduct of this study; A.F. reports grants from the NIHR, MRC, and CRUK during the conduct of this study, R.R. reports that be was partly funded by the National Health Service (NHS) (Heart of England NHS Foundation Trust) during the conduct of this study. All other authors report none.

\section{CORRESPONDING AUTHOR}

Jason $\mathrm{Oke}, \mathrm{PhD}$

Nuffield Department of Primary Care Health Sciences

University of Oxford

Radcliffe Primary Care Building

Radcliffe Observatory Quarter

Woodstock Road, Oxford OX2 6GG

jason.oke@phc.ox.ac.uk

\begin{abstract}
PURPOSE Smoking cessation after a diagnosis of lung, bladder, and upper aerodigestive tract cancer appears to improve survival, and support to quit would improve cessation. The aims of this study were to assess how often general practitioners provide active smoking cessation support for these patients and whether physician behavior is influenced by incentive payments.
\end{abstract}

METHODS Using electronic primary care records from the UK Clinical Practice Research Datalink, 12,393 patients with incident cases of cancer diagnosed between 1999 and 2013 were matched 1 to 1 to patients with incident cases of coronary heart disease (CHD) diagnosed during the same time. We assessed differences in the proportion for whom physicians updated smoking status, advised quitting, and prescribed cessation medications, as well as the proportion of patients who stopped smoking within a year of diagnosis. We further examined whether any differences arose because the physicians were offered incentives to address smoking in patients with CHD and not cancer.

RESULTS At diagnosis, $32.0 \%$ of patients with cancer and $18.2 \%$ of patients with CHD smoked tobacco. Patients with cancer were less likely than patients with CHD to have their general practitioners update smoking status (OR $=0.18 ; 95 \%$ $\mathrm{Cl}, 0.17-0.19)$, advise quitting $(\mathrm{OR}=0.38 ; 95 \% \mathrm{Cl}, 0.36-0.40)$, or prescribe medication $(\mathrm{OR}=0.67 ; 95 \% \mathrm{Cl}, 0.63-0.73)$, and they were less likely to have stopped smoking (OR $=0.76 ; 95 \% \mathrm{Cl}, 0.69-0.84)$. One year later $61.7 \%$ of patients with cancer and 55.4\% with CHD who were smoking at diagnosis were still smoking. Introducing incentive payments was associated with more frequent interventions, but not for patients with CHD specifically.

CONCLUSIONS General practitioners were less likely to support smoking cessation in patients with cancer than with CHD, and patients with cancer were less likely to stop smoking. This finding is not due to the difference in incentive payments.

Ann Fam Med 2017;15:443-450. https://doi.org/10.1370/afm.2100.

\section{INTRODUCTION}

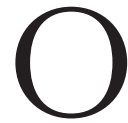
ne-fifth of cancers in the United Kingdom are attributable to tobacco smoke, ${ }^{1}$ of which cancers of the lung, bladder, and upper aerodigestive tract (combined organs and tissues of the respiratory tract and upper part of the digestive tract, including the lips, mouth, tongue, nose, throat, vocal cords, and part of the esophagus and windpipe) are most common. ${ }^{1,2}$ Continued smoking after diagnosis of cancer is associated with worse prognosis. ${ }^{3-5}$ Estimates suggest that between $35 \%$ and $62 \%$ of cancer patients continue smoking in the year after diagnosis. ${ }^{6-11}$

A cancer diagnosis motivates patients to attempt to quit smoking. ${ }^{12}$ Physicians can improve motivation and the likelihood of achieving abstinence by offering assistance, including giving advice and prescribing medication. ${ }^{13,14}$ Many physicians, however, do not view supporting smoking cessation as a priority. ${ }^{15}$ In 2004 the United Kingdom introduced a pay-for-performance scheme, the Quality and Outcomes Framework (QOF), which includes incentives to support smoking cessation. Payments are made for recording smoking status and offering support and treat- 
ment annually to patients with 1 of several smokingrelated conditions, but these conditions do not include smoking-related cancers. ${ }^{16}$ It is not known to what extent general practitioners are supporting cancer patients to quit or whether introduction of incentives for other conditions has influenced smoking cessation support for cancer patients.

The first aim of this study was to examine how often general practitioners intervene to support smoking cessation in patients with cancer, meaning how often they updated smoking status and provided support, and to examine the proportion of patients that manage to stop in the first year after diagnosis. Hospital physicians infrequently offer active support for smoking cessation, so primary care support is crucial. ${ }^{17-20}$ For context, we compared patients who had cancer with patients who had coronary heart disease (CHD), a similarly serious smoking-related condition that also motivates patients to try to stop smoking and for which there is evidence that smoking cessation improves disease outcomes. ${ }^{21} \mathrm{~A}$ second aim was to examine the effect of incentive payments on management of smoking in patients with CHD. CHD is a condition for which physicians are offered incentives to provide smoking cessation support. If management of smoking improved in patients with CHD, but not in those with cancer, after the payments were introduced, this finding would support extending incentive payments to cover patients with smoking-related cancers.

\section{METHODS}

We conducted a retrospective cohort study using routinely collected UK primary care records from the Clinical Practice Research Datalink (http://www.cprd. com). In 2013 this datalink contained records from 4.4 million live patients, $6.9 \%$ of the UK population. ${ }^{22}$ The protocol was peer reviewed then approved by the Independent Scientific Advisory Committee for Medicines and Healthcare Products Regulatory Agency database research (reference No. 14_105) and was available during peer review.

Patients with incident cases of lung, bladder, and upper aerodigestive tract cancers diagnosed between 1999 and 2013 who had a record of smoking at diagnosis or within 3 years of diagnosis were matched 1 to 1 to patients with incident CHD diagnosed during the same period as control cases based on year of diagnosis, general practice, and smoking status. We included patients who smoked at diagnosis or who had stopped within 3 years of diagnosis, because those who have recently stopped are vulnerable to relapse during this time, ${ }^{23}$ and physicians are given incentives by QOF to ask patients about smoking for up to 3 years after quit- ting. We defined smoking at diagnosis as smoking on the last occasion smoking status was recorded in the 3 years before diagnosis. A recent ex-smoker was defined as someone recorded as smoking within 3 years of diagnosis and subsequently recorded as not smoking on the last occasion before diagnosis. Patients' data were collected until the end of 2013. We adapted the protocol to exclude thyroid cancers, because they are not smoking related, and to exclude patients who had stopped smoking for more than 3 years or who had never smoked, because they were not relevant to the study questions.

For the first aim, we collected for comparison the proportion of current smokers and recent ex-smokers for whom their general practitioners updated smoking status, advised patients to stop or provided advice on how to do so, and prescribed cessation medication, as well as of patients who quit smoking during the year after diagnosis. Using logistic regression, we examined differences in outcomes between patients with cancer and control patients with CHD. All models were adjusted for age, sex, and the presence of comorbidity for which the QOF offered physicians incentives for smoking cessation support: asthma, chronic kidney disease, chronic obstructive pulmonary disease, peripheral artery disease, diabetes, hypertension, stroke, and serious mental illness. In sensitivity analyses we excluded patients who were not smoking at diagnosis. Because the physicians may not intervene on smoking in patients who are known to be terminally ill, we also conducted sensitivity analyses restricted to patients who survived at least 1 year after diagnosis. We calculated the adjusted incidence rate ratio (RR) for cancer patients relative to CHD patients for number of smoking cessation prescriptions given using negative binomial regression (to account for overdispersion). We assessed whether incentives increased the frequency of physician interventions by adding a binary term reflecting whether the year of diagnosis was before or after 2004, the year incentives were introduced. We added a multiplicative interaction term to examine whether the apparent effect of incentives differed between cancer patients and control CHD patients; the latter attracted incentive payments.

\section{RESULTS}

There were 42,112 patients who had lung, bladder, or upper aerodigestive tract cancer diagnosed between the start of 1999 and end of 2013. Of these, 13,449 (32.0\%) were smoking at diagnosis, and 3,092 (7.3\%) had stopped smoking within 3 years of diagnosis. There were 159,182 patients with CHD diagnosed during this period, of whom 28,987 (18.2\%) smoked at diagnosis and $6,301(4.0 \%)$ had stopped smoking within 3 years 
of diagnosis. Of these groups, 12,393 cancer patients were successfully matched to the same number of $\mathrm{CHD}$ control patients and were included in the main analyses. There were 9,347 patients with lung cancer $(86 \%$ current smokers), 2,050 with bladder cancer (90\% current smokers), and 996 with upper aerodigestive tract cancers (91\% current smokers). Sensitivity analyses of patients who had survived for at least 1 year included 5,094 patients with incident cases of cancer $(2,781$ lung, 1,512 bladder, 801 upper aerodigestive tract) and 5,094 matched patients with incident cases of CHD.

Cancer patients were older at diagnosis $(67.5 \mathrm{y})$ compared with CHD patients (61.3 y), were less likely to be male $(57.9 \%$ vs $65.6 \%$, respectively), and had higher prevalences of asthma, chronic obstructive pulmonary disease, chronic kidney disease, stroke, and peripheral artery disease and lower prevalences of hypertension and diabetes (Table 1).

\section{Updating of Smoking Status}

Cancer patients were significantly less likely to have their smoking status updated during the first year after diagnosis than control patients (37\% vs $78 \%$ ). After removing patients who died within a year of diagnosis, this difference was smaller but still apparent $(62 \%$ vs $86 \%$ ) (Table 2, Figure 1a).

There was an almost 3 -fold increase in the odds of updating smoking status after incentives were introduced $(\mathrm{OR}=2.71 ; 95 \% \mathrm{CI}, 2.44-2.99)$. There was no evidence that the increase was larger in CHD patients compared with cancer patients $(P=.86)$ (Supplemental Table 1, http://www.annfammed.org/content/15/5/443/ suppl/DC1).

\section{Advice to Quit}

Cancer patients were significantly less likely to have a recording of advice to quit (all patients, $\mathrm{OR}=0.38$, (95\% CI, 0.36-0.40)). When including patients who were smoking at diagnosis only, the odds ratio was similar, $\mathrm{OR}=0.36(95 \% \mathrm{CI}, 0.34-0.38))$. In the cohort that survived at least 1 year, the odds ratio was 0.60 , (95\% CI, 0.55-0.66) (Table 2, Figure 1b).

There was a 3 -fold increase in the odds of recording advice to quit after the introduction of incentives $(\mathrm{OR}=3.04 ; 95 \% \mathrm{CI}, 2.73-3.38)$. There was evidence that the increase in odds was greater for cancer patients than for CHD control patients $(P=.02)$, and subgroup analyses showed that increased odds were confined to lung cancer patients (Supplemental Table 2, http:// www.annfammed.org/content/15/5/443/suppl/DC1).

\section{Prescription of Smoking Cessation Medications}

Cancer patients were significantly less likely to be prescribed smoking cessation medications (all patients,
Table 1. Distribution of Baseline Characteristics in All Patients With Cancer Matched to Control Patients With CHD, Diagnosed Between 1999 and 2013

\begin{tabular}{|c|c|c|}
\hline Characteristic & $\begin{array}{c}\text { All Cancer } \\
\text { Patients } \\
\mathbf{n}=12,393\end{array}$ & $\begin{array}{c}\text { CHD Patients } \\
n=12,393\end{array}$ \\
\hline \multicolumn{3}{|l|}{ Sex, No. (\%) } \\
\hline Male & 7,185 (57.9) & $8,126(65.6)$ \\
\hline Female & $5,208(42.1)$ & $4,267(34.4)$ \\
\hline Age, mean (SD), y & $67.5(10.5)$ & $61.3(11.9)$ \\
\hline \multicolumn{3}{|l|}{$\begin{array}{l}\text { Smoking status at diag- } \\
\text { nosis, No. (\%) }\end{array}$} \\
\hline Current & $10,794(87.1)$ & $10,794(87.1)$ \\
\hline$<3$-y ex-smoker & $1,599(12.9)$ & $1,599(12.9)$ \\
\hline \multicolumn{3}{|l|}{$\begin{array}{l}\text { Index of multiple depri- } \\
\text { vation, No. (\%) }\end{array}$} \\
\hline 1 (least deprived) & $925(7.5)$ & $934(7.5)$ \\
\hline 2 & $1,315(10.6)$ & $1,372(11.1)$ \\
\hline 3 & $1,423(11.5)$ & $1,414(11.4)$ \\
\hline 4 & $1,758(14.2)$ & $1,690(13.6)$ \\
\hline 5 (most deprived) & $1,764(14.2)$ & $1,773(14.3)$ \\
\hline Missing & $5,208(42.0)$ & $5,210(42.0)$ \\
\hline \multicolumn{3}{|l|}{$\begin{array}{l}\text { Frequency of smoking, } \\
\text { No. (\%) }\end{array}$} \\
\hline Light & $1,496(13.9)$ & $1,367(12.7)$ \\
\hline Moderate & $2,093(19.4)$ & $1,981(18.4)$ \\
\hline Heavy & $1,788(16.6)$ & 1,794 (16.6) \\
\hline Frequency unknown & $5,417(50.2)$ & $5,652(52.4)$ \\
\hline \multicolumn{3}{|l|}{ Asthma, No. (\%) } \\
\hline No & 11,271 (91.9) & $11,456(92.4)$ \\
\hline Yes & $1122(9.1)$ & $937(7.6)$ \\
\hline \multicolumn{3}{|l|}{$\begin{array}{l}\text { Chronic kidney disease, } \\
\text { No. (\%) }\end{array}$} \\
\hline No & $11,481(92.6)$ & 11,767 (94.9) \\
\hline Yes & $912(7.4)$ & $626(5.1)$ \\
\hline \multicolumn{3}{|l|}{$\begin{array}{l}\text { Chronic obstructive } \\
\text { pulmonary disease, } \\
\text { No. (\%) }\end{array}$} \\
\hline No & $9,642(77.8)$ & $11,091(89.5)$ \\
\hline Yes & $2751(22.2)$ & $1302(10.5)$ \\
\hline \multicolumn{3}{|l|}{ Diabetes, No. (\%) } \\
\hline No & 11,339 (91.5) & $11,182(90.2)$ \\
\hline Yes & $1054(8.5)$ & $1211(9.8)$ \\
\hline \multicolumn{3}{|l|}{ Hypertension, No. (\%) } \\
\hline No & $9,887(79.8)$ & $9,658(77.9)$ \\
\hline Yes & $2506(20.2)$ & $2735(22.1)$ \\
\hline \multicolumn{3}{|l|}{$\begin{array}{l}\text { Peripheral arterial } \\
\text { disease, No. (\%) }\end{array}$} \\
\hline No & $11,517(92.9)$ & $11,649(94)$ \\
\hline Yes & $876(7.1)$ & $744(6.0)$ \\
\hline \multicolumn{3}{|l|}{ Stroke, No. (\%) } \\
\hline No & $11,582(93.5)$ & $11,790(95.1)$ \\
\hline Yes & $811(6.5)$ & $603(4.9)$ \\
\hline \multicolumn{3}{|l|}{ Psychosis, No. (\%) } \\
\hline No & $12,289(99.2)$ & $12,306(99.3)$ \\
\hline Yes & $104(0.8)$ & $87(0.7)$ \\
\hline
\end{tabular}




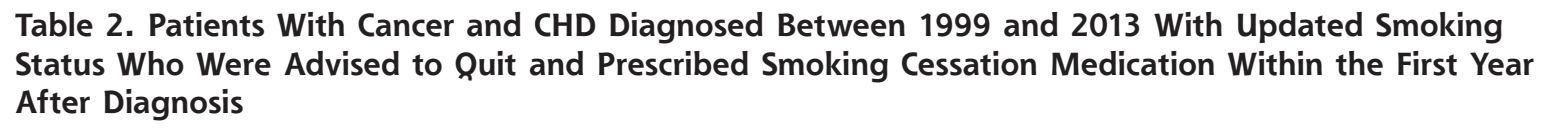

\begin{tabular}{|c|c|c|c|c|c|c|c|c|c|}
\hline \multirow[b]{2}{*}{ Outcome } & \multicolumn{3}{|c|}{$\begin{array}{c}\text { Smokers and } \\
<3-y \text { Ex-Smokers } \\
\text { (All Cancer, } \mathrm{n}=12,393 ; \\
\text { CHD, } \mathrm{n}=12,393)^{\mathrm{a}}\end{array}$} & \multicolumn{3}{|c|}{$\begin{array}{l}\text { Current Smokers Only } \\
\text { (All Cancer, } n=10,794 ; \\
\text { CHD, } n=10,794)^{b}\end{array}$} & \multicolumn{3}{|c|}{$\begin{array}{l}\geq 1-y \text { Survivors Only } \\
\text { (All Cancer, } n=4,228 ; \\
\text { CHD, } n=4,228)^{c}\end{array}$} \\
\hline & $\begin{array}{l}\text { Cancer } \\
\text { No. (\%) }\end{array}$ & $\begin{array}{l}\text { CHD } \\
\text { Control } \\
\text { No. }(\%)\end{array}$ & $\begin{array}{c}\text { OR } \\
(95 \% \mathrm{Cl})\end{array}$ & $\begin{array}{l}\text { Cancer } \\
\text { No. (\%) }\end{array}$ & $\begin{array}{l}\text { CHD } \\
\text { Control } \\
\text { No. (\%) }\end{array}$ & $\begin{array}{c}\text { OR } \\
(95 \% \mathrm{Cl})\end{array}$ & $\begin{array}{l}\text { Cancer } \\
\text { No. (\%) }\end{array}$ & $\begin{array}{l}\text { CHD } \\
\text { Control } \\
\text { No. (\%) }\end{array}$ & $\begin{array}{c}\text { OR } \\
(95 \% \mathrm{Cl})\end{array}$ \\
\hline \multicolumn{10}{|c|}{ Updated smoking status } \\
\hline All cancers & $\begin{array}{c}4,541 \\
(37)\end{array}$ & $\begin{array}{c}9,627 \\
(78)\end{array}$ & $\begin{array}{c}0.18 \\
(0.17-0.19)\end{array}$ & $\begin{array}{c}3,962 \\
(37)\end{array}$ & $\begin{array}{c}8,437 \\
(78)\end{array}$ & $\begin{array}{c}0.18 \\
(0.17-0.19)\end{array}$ & $\begin{array}{c}2,605 \\
(62)\end{array}$ & $\begin{array}{c}3,611 \\
(86)\end{array}$ & $\begin{array}{c}0.26 \\
(0.23-0.29)\end{array}$ \\
\hline Lung cancer & $\begin{array}{c}2,873 \\
(31)\end{array}$ & $\begin{array}{c}7,224 \\
(77)\end{array}$ & $\begin{array}{c}0.14 \\
(0.13-0.15)\end{array}$ & $\begin{array}{c}2,454 \\
(31)\end{array}$ & $\begin{array}{c}6,253 \\
(78)\end{array}$ & $\begin{array}{c}0.13 \\
(0.12-0.14)\end{array}$ & $\begin{array}{l}1,404 \\
(60)\end{array}$ & $\begin{array}{c}1,982 \\
(84)\end{array}$ & $\begin{array}{c}0.25 \\
(0.22-0.29)\end{array}$ \\
\hline Bladder cancer & $\begin{array}{l}1,172 \\
(57)\end{array}$ & $\begin{array}{c}1,620 \\
(79)\end{array}$ & $\begin{array}{c}0.38 \\
(0.33-0.44)\end{array}$ & $\begin{array}{l}1,055 \\
(57)\end{array}$ & $\begin{array}{c}1,466 \\
(79)\end{array}$ & $\begin{array}{c}0.38 \\
(0.33-0.44)\end{array}$ & $\begin{array}{l}851 \\
(65)\end{array}$ & $\begin{array}{l}1,138 \\
(87)\end{array}$ & $\begin{array}{c}0.28 \\
(0.22-0.34)\end{array}$ \\
\hline $\begin{array}{l}\text { Upper aerodigestive } \\
\text { tract cancer }\end{array}$ & $\begin{array}{l}496 \\
(50)\end{array}$ & $\begin{array}{l}783 \\
(79)\end{array}$ & $\begin{array}{c}0.27 \\
(0.22-0.33)\end{array}$ & $\begin{array}{l}453 \\
(50)\end{array}$ & $\begin{array}{l}718 \\
(79)\end{array}$ & $\begin{array}{c}0.27 \\
(0.22-0.33)\end{array}$ & $\begin{array}{l}350 \\
(61)\end{array}$ & $\begin{array}{l}502 \\
(87)\end{array}$ & $\begin{array}{c}0.23 \\
(0.17-0.30)\end{array}$ \\
\hline \multicolumn{10}{|l|}{ Advice to quit } \\
\hline All cancers & $\begin{array}{c}2,794 \\
(23)\end{array}$ & $\begin{array}{c}5,601 \\
(45)\end{array}$ & $\begin{array}{c}0.38 \\
(0.36-0.40)\end{array}$ & $\begin{array}{c}2,636 \\
(24)\end{array}$ & $\begin{array}{c}5,245 \\
(48)\end{array}$ & $\begin{array}{c}0.36 \\
(0.34-0.38)\end{array}$ & $\begin{array}{l}1,630 \\
(39)\end{array}$ & $\begin{array}{c}2,156 \\
(51)\end{array}$ & $\begin{array}{c}0.60 \\
(0.55-0.66)\end{array}$ \\
\hline Lung cancer & $\begin{array}{c}1,672 \\
(18)\end{array}$ & $\begin{array}{c}4,196 \\
(45)\end{array}$ & $\begin{array}{c}0.28 \\
(0.26-0.30)\end{array}$ & $\begin{array}{c}1,564 \\
(19)\end{array}$ & $\begin{array}{c}3,907 \\
(49)\end{array}$ & $\begin{array}{c}0.26 \\
(0.24-0.28)\end{array}$ & $\begin{array}{l}810 \\
(34)\end{array}$ & $\begin{array}{c}1,182 \\
(50)\end{array}$ & $\begin{array}{c}0.49 \\
(0.43-0.56)\end{array}$ \\
\hline Bladder cancer & $\begin{array}{l}809 \\
(39)\end{array}$ & $\begin{array}{l}925 \\
(45)\end{array}$ & $\begin{array}{c}0.87 \\
(0.76-0.99)\end{array}$ & $\begin{array}{l}774 \\
(42)\end{array}$ & $\begin{array}{l}880 \\
(48)\end{array}$ & $\begin{array}{c}0.86 \\
(0.75-0.98)\end{array}$ & $\begin{array}{l}594 \\
(46)\end{array}$ & $\begin{array}{l}669 \\
(51)\end{array}$ & $\begin{array}{c}0.84 \\
(0.70-0.99)\end{array}$ \\
\hline $\begin{array}{l}\text { Upper aerodigestive } \\
\text { tract cancer }\end{array}$ & $\begin{array}{l}313 \\
(31)\end{array}$ & $\begin{array}{l}480 \\
(48)\end{array}$ & $\begin{array}{c}0.50 \\
(0.41-0.60)\end{array}$ & $\begin{array}{l}298 \\
(33)\end{array}$ & $\begin{array}{l}458 \\
(50)\end{array}$ & $\begin{array}{c}0.50 \\
(0.41-0.60)\end{array}$ & $\begin{array}{l}226 \\
(39)\end{array}$ & $\begin{array}{l}305 \\
(53)\end{array}$ & $\begin{array}{c}0.58 \\
(0.46-0.74)\end{array}$ \\
\hline \multicolumn{10}{|l|}{ Prescriptions } \\
\hline All cancers & $\begin{array}{l}1,504 \\
(12)\end{array}$ & $\begin{array}{c}2,560 \\
(21)\end{array}$ & $\begin{array}{c}0.67 \\
(0.63-0.73)\end{array}$ & $\begin{array}{c}1,439 \\
(13)\end{array}$ & $\begin{array}{c}2,426 \\
(22)\end{array}$ & $\begin{array}{c}0.67 \\
(0.62-0.72)\end{array}$ & $\begin{array}{l}882 \\
(21)\end{array}$ & $\begin{array}{l}967 \\
(23)\end{array}$ & $\begin{array}{c}1.05 \\
(0.94-1.17)\end{array}$ \\
\hline Lung cancer & $\begin{array}{l}989 \\
(11)\end{array}$ & $\begin{array}{l}1,950 \\
(21)\end{array}$ & $\begin{array}{c}0.58 \\
(0.53-0.63)\end{array}$ & $\begin{array}{l}940 \\
(12)\end{array}$ & $\begin{array}{l}1,835 \\
(23)\end{array}$ & $\begin{array}{c}0.57 \\
(0.52-0.63)\end{array}$ & $\begin{array}{l}498 \\
(21)\end{array}$ & $\begin{array}{l}547 \\
(23)\end{array}$ & $\begin{array}{c}1.06 \\
(0.91-1.23)\end{array}$ \\
\hline Bladder cancer & $\begin{array}{l}291 \\
(14)\end{array}$ & $\begin{array}{l}386 \\
(19)\end{array}$ & $\begin{array}{c}0.96 \\
(0.81-1.16)\end{array}$ & $\begin{array}{l}279 \\
(15)\end{array}$ & $\begin{array}{l}373 \\
(20)\end{array}$ & $\begin{array}{c}0.97 \\
(0.81-1.16)\end{array}$ & $\begin{array}{l}226 \\
(17)\end{array}$ & $\begin{array}{l}280 \\
(22)\end{array}$ & $\begin{array}{c}1.02 \\
(0.83-1.25)\end{array}$ \\
\hline $\begin{array}{l}\text { Upper aerodigestive } \\
\text { tract cancer }\end{array}$ & $\begin{array}{l}224 \\
(22)\end{array}$ & $\begin{array}{l}224 \\
(22)\end{array}$ & $\begin{array}{c}1.00 \\
(0.80-1.24)\end{array}$ & $\begin{array}{l}220 \\
(24)\end{array}$ & $\begin{array}{l}218 \\
(24)\end{array}$ & $\begin{array}{c}1.03 \\
(0.83-1.29)\end{array}$ & $\begin{array}{l}158 \\
(27)\end{array}$ & $\begin{array}{l}140 \\
(24)\end{array}$ & $\begin{array}{c}1.18 \\
(0.89-1.55)\end{array}$ \\
\hline \multicolumn{10}{|c|}{$\mathrm{CHD}=$ coronary heart disease; $\mathrm{UAT}=$ upper aeordigestive tract. } \\
\hline \multicolumn{10}{|c|}{$\begin{array}{l}\text { a Cancer subgroup patients and 1-to-1 matched CHD control patients: lung, } n=9,347 ; \text { bladder, } n=2,050 ; \text { UAT, } n=996 \text {. } \\
\text { b Cancer subgroup patients and 1-to-1 matched CHD control patients: lung, } n=8,037 ; \text { bladder, } n=1,848 ; \text { UAT, } n=909 \text {. } \\
\text { 'Cancer subgroup patients and 1-to-1 matched CHD control patients: lung, } n=2,350 ; \text { bladder, } n=1,302 ; \text { UAT, } n=576 \text {. }\end{array}$} \\
\hline
\end{tabular}

$\mathrm{OR}=0.67,(95 \% \mathrm{CI}, 0.63-0.73)$, and current smokers at diagnosis only, $\mathrm{OR}=0.67,(95 \% \mathrm{CI}, 0.62-0.72)$. This difference was smaller, however, not significant, and confined to patients who survived at least a year $\mathrm{OR}=1.05$, (95\% CI, 0.94-1.17) (Table 2, Figure 1c). The number of prescriptions given to cancer patients was similar to the number given to CHD control patients, $\mathrm{RR}=0.95$ (95\% CI, 0.87-1.04). Restricted to those surviving a year it was 1.15 (95\% CI, 1.01-1.32) indicating that cancer patients were given more prescriptions than CHD control patients.

There was a significant increase in proportion of patients receiving smoking cessation medications after introduction of the QOF $(\mathrm{OR}=1.79 ; 95 \% \mathrm{CI}, 1.56$ 2.05). There was no evidence that this change in the odds of prescribing at least 1 medication differed for cancer or CHD patients $(P=.89)$. Findings were similar in cancer subgroups matched to $\mathrm{CHD}$ control patients (Supplemental Table 3, http://www.annfammed.org/ content/15/5/443/suppl/DC1).

\section{Smoking Cessation}

Of the 3,706 cancer and CHD patients who smoked at diagnosis and had at least 1 smoking status update in the year following diagnosis, $1,359(36.7 \%)$ of patients with cancer and 1,645 (44.4\%) of patients with CHD stopped smoking. Among 2,253 pairs, both of whom had smoking status updated and survived at least 1 year, $863(38.3 \%)$ with cancer and 1,004 (44.6\%) with CHD stopped smoking (Table 3). 
There was no significant increase in quitting after introduction of incentives $(\mathrm{OR}=1.18$; 95\% CI, 0.94-1.49) (Supplemental Table 4, http://www.annfammed.org/content/15/5/443/ suppl/DC1).

\section{DISCUSSION}

One-third of patients with lung, bladder, and upper aerodigestive tract cancer smoked at diagnosis. Patients with cancer were less likely to have smoking status recorded by their general practitioner, be given advice, be prescribed cessation pharmacotherapy, or quit smoking in the year following diagnosis. Confining the analysis to patients who smoked at the time of diagnosis and to those with a better prognosis did not change these findings, except that the difference in prescription of pharmacotherapy was no longer apparent. The frequency of recording of smoking status, advice, and pharmacotherapy increased after introduction of incentive payments for physicians to manage smoking, but there were no differences in the rates of quitting. As these payments were confined to the management of smoking in patients with CHD and not cancer, we expected to see the improvement to be larger in the CHD group; there was no evidence of such an improvement, however, and some evidence of the reverse.

\section{Strengths and Weaknesses}

This study is the first to investigate how general practitioners manage smoking in patients with smoking-related cancer. An important strength is that the population of patients and physicians who provide data to CPRD is broadly representa-
Figure 1. Percentage of patients who had smoking status updated, advice to quit, and prescription of smoking cessation medications, and who quit within the first year after diagnosis before and after QOF (all cancer patients and matched CHD patients) between 1999 and 2013.

A. Smoking status (pre QOF/post QOF: OR $=2.71(95 \% \mathrm{Cl}, 2.44-2.99), P=.86$

$$
\text { CHD controls (\%) - Cancer cases (\%) }
$$

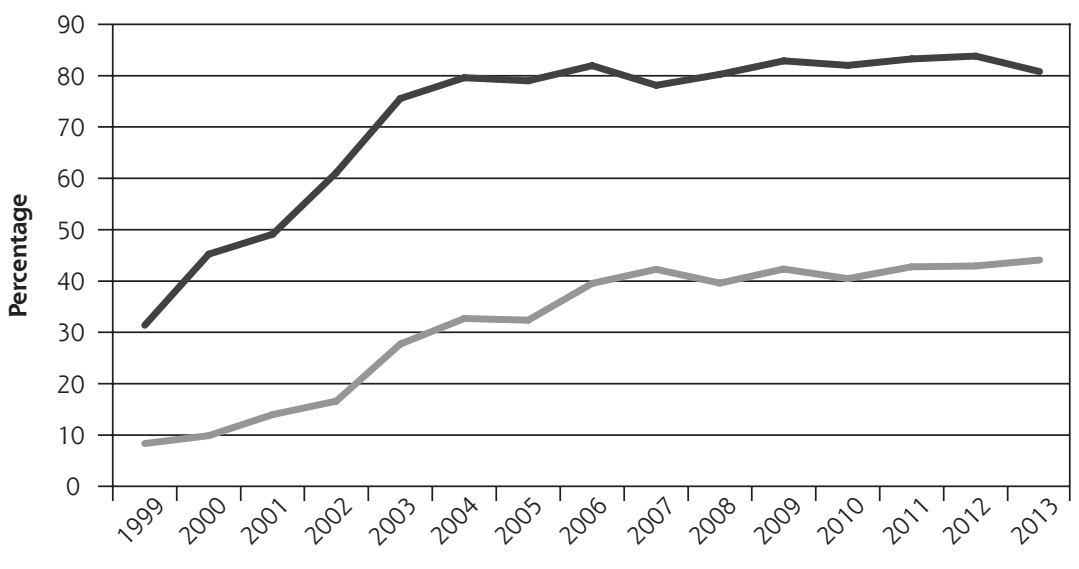

B. Advice to quit (pre QOF/post QOF: OR $=3.04$ (95\% Cl, 2.73-3.38), $P=.02$

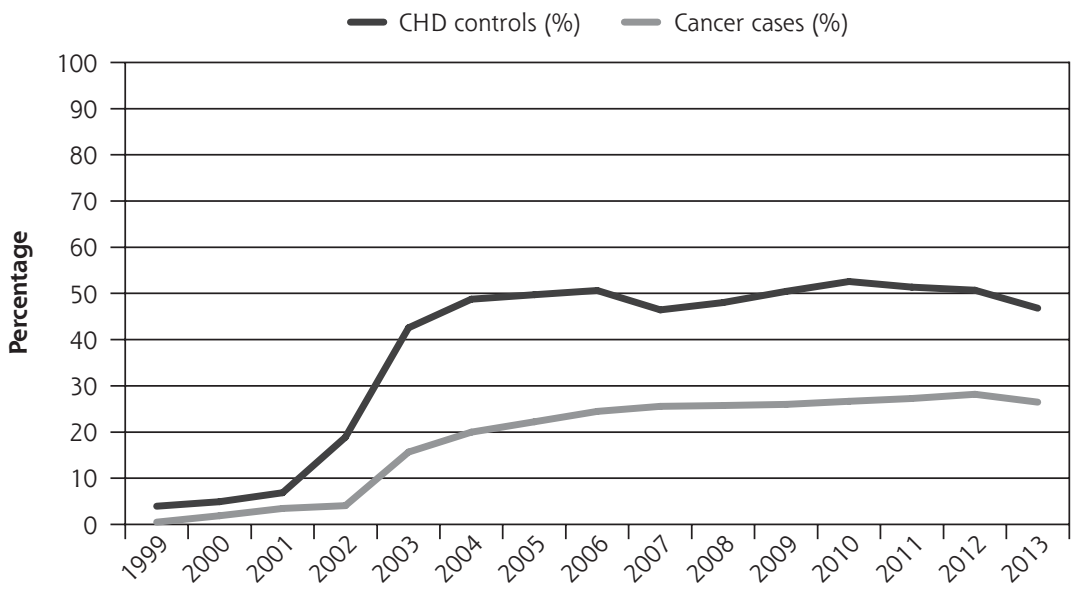

C. Prescriptions (pre QOF/post QOF: OR $=1.79$ (95\% Cl, 1.56-2.05), $P=.89$

$\longrightarrow$ CHD controls (\%) Cancer cases (\%)

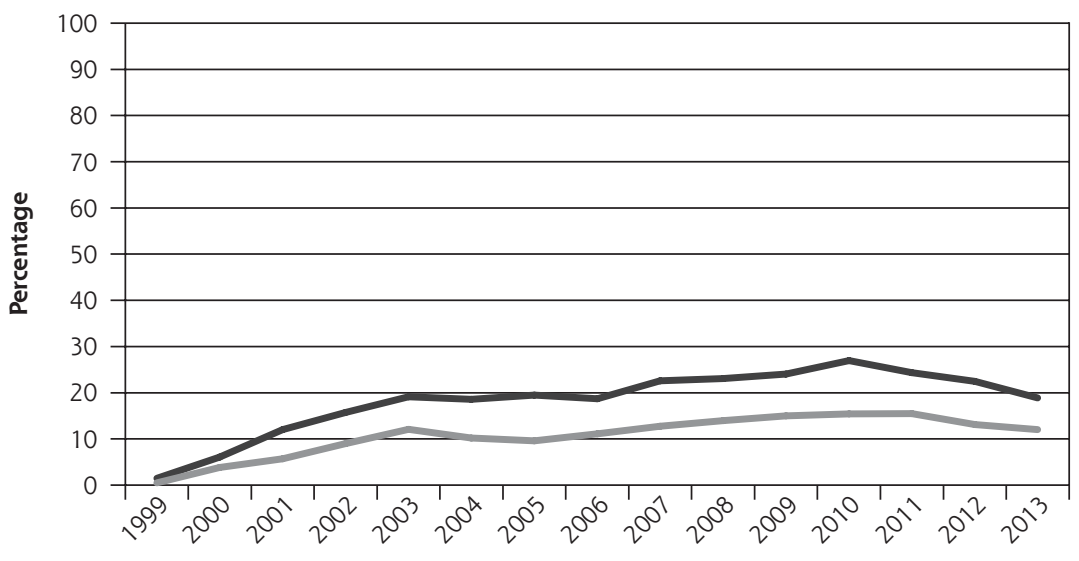

$\mathrm{CHD}=$ coronary heart disease; $\mathrm{OR}=$ odds ratio; $\mathrm{QOF}=$ Quality and Outcomes Framework 


\section{Table 3. Patients With Cancer and CHD Diagnosed Between 1999 and 2013,} Quitting Within the First Year After Diagnosis

\begin{tabular}{|c|c|c|c|c|c|c|}
\hline \multirow[b]{2}{*}{ Quitting } & \multicolumn{3}{|c|}{$\begin{array}{l}\text { Patients With } \geq 1 \text { Update of Smoking Status } \\
\text { (All Cancer, } n=3,706 ; \text { CHD Control, } n=3,706)^{\text {a }}\end{array}$} & \multicolumn{3}{|c|}{$\begin{array}{c}\text { Patients With } \geq 1 \text { Update of } \\
\text { Smoking Status and } \geq 1-y \text { Survivors Only } \\
\text { (All Cancer, } n=2,253 \text {; CHD Control, } n=2,253)^{b}\end{array}$} \\
\hline & $\begin{array}{l}\text { Cancer } \\
\text { No. (\%) }\end{array}$ & $\begin{array}{l}\text { CHD Control } \\
\text { No. (\%) }\end{array}$ & OR $(95 \% \mathrm{Cl})$ & $\begin{array}{l}\text { Cancer } \\
\text { No. (\%) }\end{array}$ & $\begin{array}{l}\text { CHD Control } \\
\text { No. }(\%)\end{array}$ & OR $(95 \% \mathrm{Cl})$ \\
\hline All cancers & $1,359(36.7)$ & $1,645(44.4)$ & $0.76(0.69-0.84)$ & $863(38.3)$ & $1,004(44.6)$ & $0.82(0.72-0.93)$ \\
\hline Lung cancer & $885(37.8)$ & $1,019(43.6)$ & $0.85(0.75-0.97)$ & $487(41.3)$ & $510(43.3)$ & $1.04(0.87-1.25)$ \\
\hline Bladder cancer & 289 (30.6) & $445(47.1)$ & $0.48(0.39-0.59)$ & $232(30.7)$ & 351 (46.5) & $0.50(0.40-0.63)$ \\
\hline $\begin{array}{l}\text { Upper aerodigestive } \\
\text { tract cancer }\end{array}$ & $185(43.8)$ & $181(42.9)$ & $1.03(0.78-1.35)$ & $144(45.0)$ & $143(44.7)$ & $1.00(0.73-1.38)$ \\
\hline
\end{tabular}

tive of the general UK population. ${ }^{22}$ The sample was large enough to give precise estimates of association. Similar to all observational studies, we are unable to conclude that the lower rates of physician intervention on smoking in cancer patients were due to the physician not prioritizing smoking in this group specifically. One plausible explanation could be that patients with cancer were less likely to consult general practitioners than were patients with $\mathrm{CHD}$, although there was no evidence of this likelihood. Ninety-one percent of all patients with a new diagnosis of cancer were seen by their general practitioner in the year after diagnosis, as were $95 \%$ of all cancer patients who survived at least 1 year, compared with $75 \%$ and $79 \%$ of patients with CHD. Another explanation could lie in differences in expected survival between patients with lung cancer in particular and patients with CHD. Arguably, it is inappropriate for general practitioners to intervene on smoking in patients with only months to live, and many patients with lung cancer diagnosed survive for less than a year. ${ }^{24}$ To see whether difference in management was driven by expectations of poor prognosis, we undertook a sensitivity analyses using only patients that survived 1 year. We had originally planned to assess the effect of expected prognosis by adjusting for treatment intent and cancer staging at diagnosis, but the data were not available in the level of Hospital Episode Statistics to which we had access. Limiting analysis to patients who survived at least 1 year narrowed but generally did not abolish the difference in smoking management between patients with cancer and CHD. An additional reason for lower physician intervention may be that cancer patients are more likely to report receiving help from secondary care. Support for smoking cessation in secondary care is low, however, and it is unlikely to be the main source of cessation support. ${ }^{17-20}$

As with all studies based on health care records, it is possible that the general practitioners provided advice to quit or on how to quit which they did not record. Although underreporting would underestimate the true rate of intervention, it is likely to underestimate the frequency of intervention in patients with cancer and CHD equally and is thus an implausible explanation for the findings. Given the way records work, all prescriptions given by general practitioners would have been recorded, and thus these data can be regarded as true estimates of the frequency of intervention. It is also likely that some patients stopped smoking, which, because the physicians did not ask, was not recorded, or patients may have claimed to have stopped smoking when such was not the case. Even though our estimate of cessation may therefore be inaccurate to some degree, any error should affect patients with cancer and CHD similarly. Thus it appears that general practitioners are less assiduously supporting patients with cancer to stop smoking than they are for patients with CHD to the detriment of patients with cancer.

\section{Interpretation of Findings and Comparison With Existing Studies}

Many general practitioners express negative attitudes toward supporting smoking cessation in general, ${ }_{1}^{15}$ including concerns about lack of time, ineffectiveness of interventions, and lack of training. These concerns would deter intervening in patients with lung cancer and CHD equally, however. Two recent international surveys of cancer specialists found that less than one-half routinely offered patients smoking cessation treatment; common concerns were that intervention would be ineffective, and cancer patients would resist treatment. ${ }^{18,19}$ Even so, we have previously reported that patients treated surgically for lung cancer express a wish for much greater involvement of clinicians 
in helping them manage smoking, so this concern may be unfounded. ${ }^{25}$ Whether general practitioners view smoking cessation support as an equal priority is perhaps a secondary concern, however, because data indicate considerable scope for increasing active management both in cancer and CHD patients. Only a minority of patients receive advice to quit, and just more than one-fifth are prescribed pharmacotherapy, whereas about 6 in 10 report smoking 1 year after the diagnosis of cancer or CHD.

The second aim was to examine the impact of payment to general practitioners for management of smoking. We found that recording of status and advice was higher on average in the post-QOF period than before incentives were introduced in 2004. Given the size of effect and the sharp rise that occurred around the time of introducing the incentive payments, it is likely that this change was brought about by the new payment system, as has been noted in previous studies. ${ }^{26,27}$ Counterintuitively, however, we found that the increase applied equally to patients with cancer, who did not attract payments, and to patients with CHD, who did. Furthermore, the absolute rates of prescriptions of smoking cessation medications were small, and although they increased after introduction of the QOF, the size of the increase for cancer and CHD patients was similar. Although physician intervention for smoking has been shown to be effective at increasing smoking cessation rates, ${ }^{14}$ and incentive payments will have the desired benefit to health if activity leads patients to quit smoking ${ }_{1}^{28}$ this finding casts doubt on the specific benefits of extending the coverage of incentives for patients with smoking-related cancers.

Our data show that cancer patients receive less support to quit smoking in primary care than did patients with CHD. Although absolute rates have improved with time, they remain lower than they could be. The higher rate of intervention seen in patients with CHD than with cancer is not due to the effect of incentive payments. Cancer patients would benefit if general practitioners became more actively involved in supporting smoking cessation, and it is important to find ways to improve the management of smoking cessation for patients with cancer.

To read or post commentaries in response to this article, see it online at http://www.AnnFamMed.org/content/15/5/443.

Key words: smoking; smoking cessation; cancer; primary care

Submitted October 4, 2016; submitted, revised January 27, 2017; accepted February 19, 2017.

Author contributions: A.F., P.A., R.R., and L.S. developed the study protocol which was approved by all authors. C.K., J.O., and R.S. conducted the data processing and analysis. Drafting of the manuscript was led by A.F. and contributed to by all authors. All authors approved the final version of the manuscript. R.S. and J.O. had full access to all the data in the study and take responsibility for the integrity of the data and the accuracy of the data analysis.

Funding support: This study was funded by the NIHR School for Primary Care Research (project No. 224).

Disclaimer: The funder was not involved in the design and conduct of the study; collection, management, analysis, and interpretation of the data; or preparation, review, or approval of the manuscript.

Supplementary materials: Available at http://www.AnnFamMed. org/content/15/5/443/suppl/DC1/.

\section{References}

1. Parkin DM, Boyd L, Walker LC. 16. The fraction of cancer attributable to lifestyle and environmental factors in the UK in 2010. Br J Cancer. 2011;105(Suppl 2):S77-S81.

2. Cancer Research UK. CRUK 2015 Cancer incidence for common cancers. 2015. http://www.cancerresearchuk.org/health-professional/ cancer-statistics/incidence/common-cancers-compared.

3. US Surgeon General. The Health Consequences of Smoking -50 Years of Progress: A Report of the Surgeon General. Washington, DC: US Department of Health and Human Services; 2014.

4. Parsons A, Daley A, Begh R, Aveyard P. Influence of smoking cessation after diagnosis of early stage lung cancer on prognosis: systematic review of observational studies with meta-analysis. BMJ. 2010;340:b5569.

5. Aveyard P, Adab P, Cheng KK, Wallace DM, Hey K, Murphy MF. Does smoking status influence the prognosis of bladder cancer? A systematic review. BJU Int. 2002;90(3):228-239.

6. Cooley ME, Sarna L, Kotlerman J, et al. Smoking cessation is challenging even for patients recovering from lung cancer surgery with curative intent. Lung Cancer. 2009;66(2):218-225.

7. Park ER, Japuntich SJ, Rigotti NA, et al. A snapshot of smokers after lung and colorectal cancer diagnosis. Cancer. 2012;118(12):3153-3164.

8. Sanderson Cox L, Sloan JA, Patten CA, et al. Smoking behavior of 226 patients with diagnosis of stage IIIA/IIIB non-small cell lung cancer. Psychooncology. 2002;11(6):472-478.

9. Duffy SA, Khan MJ, Ronis DL, et al. Health behaviors of head and neck cancer patients the first year after diagnosis. Head Neck. 2008;30(1):93-102.

10. Ostroff JS, Jacobsen PB, Moadel AB, et al. Prevalence and predictors of continued tobacco use after treatment of patients with head and neck cancer. Cancer. 1995;75(2):569-576.

11. Walker MS, Vidrine DJ, Gritz ER, et al. Smoking relapse during the first year after treatment for early-stage non-small-cell lung cancer. Cancer Epidemiol Biomarkers Prev. 2006;15(12):2370-2377.

12. Gritz ER, Fingeret MC, Vidrine DJ, Lazev AB, Mehta NV, Reece GP. Successes and failures of the teachable moment: smoking cessation in cancer patients. Cancer. 2006;106(1):17-27.

13. Boyle R, Solberg L, Fiore M. Use of electronic health records to support smoking cessation. Cochrane Database Syst Rev. 2014;(12): CD008743.

14. Stead LF, Buitrago D, Preciado N, Sanchez G, Hartmann-Boyce J, Lancaster T. Physician advice for smoking cessation. Cochrane Database Syst Rev. 2013;5(5):CD000165.

15. Vogt F, Hall S, Marteau TM. General practitioners' and family physicians' negative beliefs and attitudes towards discussing smoking cessation with patients: a systematic review. Addiction. 2005;100(10):1423-1431. 
16. Department of Health. Investing in General Practice: the New General Medical Services Contract. 2003. http://www.nhsemployers.org/ /media/Employers/Documents/SiteCollectionDocuments/ gms_contract_cd_130209.pdf.

17. Murray R, Leonardi-Bee J, Marsh J, Jayes L, Britton J. Smoking status ascertainment and interventions in acute medical patients. Clin Med (Lond). 2012;12(1):59-62.

18. Warren GW, Marshall JR, Cummings KM, et al.; IASLC Tobacco Control and Smoking Cessation Committee. Practice patterns and perceptions of thoracic oncology providers on tobacco use and cessation in cancer patients. J Thorac Oncol. 2013;8(5):543-548.

19. Warren GW, Marshall JR, Cummings KM, et al. Addressing tobacco use in patients with cancer: a survey of American Society of Clinical Oncology members. J Oncol Pract. 2013;9(5):258-262.

20. British Thoracic Society. British Thoracic Society recommendations for hospital smoking cessation services for commissioners and health care professionals. British Thoracic Society Reports. 2012;4(4).

21. Critchley JA, Capewell S. Mortality risk reduction associated with smoking cessation in patients with coronary heart disease: a systematic review. JAMA. 2003;290(1):86-97.

22. Herrett E, Gallagher AM, Bhaskaran K, et al. Data Resource Profile: Clinical Practice Research Datalink (CPRD). Int J Epidemiol. 2015;44(3):827-836.
23. Piasecki TM. Relapse to smoking. Clin Psychol Rev. 2006;26(2):196-215.

24. Cancer Research UK. Lung cancer survival statistics 2015. http:// www.cancerresearchuk.org/health-professional/cancer-statistics/ statistics-by-cancer-type/lung-cancer\#heading-Two.

25. Farley A, Aveyard P, Kerr A, Naidu B, Dowswell G. Surgical lung cancer patients' views about smoking and support to quit after diagnosis: a qualitative study. J Cancer Surviv. 2016;10(2):312-319.

26. Taggar JS, Coleman T, Lewis S, Szatkowski L. The impact of the Quality and Outcomes Framework (QOF) on the recording of smoking targets in primary care medical records: cross-sectional analyses from The Health Improvement Network (THIN) database. BMC Public Health. 2012;12:329.

27. Coleman T, Lewis S, Hubbard R, Smith C. Impact of contractual financial incentives on the ascertainment and management of smoking in primary care. Addiction. 2007;102(5):803-808.

28. Coleman T. Do financial incentives for delivering health promotion counselling work? Analysis of smoking cessation activities stimulated by the quality and outcomes framework. BMC Public Health. 2010;10(167):167.

\section{CHANGE-OF-ADDRESS FORM

Please complete this form and mail to the following address or fax to Annals Circulation at 913-906-6080:

Annals of Family Medicine, Circulation Department, 11400 Tomahawk Creek Pkwy, Leawood, KS 66211-2680

Check if member of sponsoring organization:

$$
\begin{array}{ll}
\square \text { AAFP } & \square \text { ABFM } \square \text { STFM } \square \text { ADFM } \\
\square \text { AFMRD } & \square \text { NAPCRG } \square \text { CFPC }
\end{array}
$$

ID number from label on your journal cover

OLD Information (Please print.)

\begin{tabular}{ll}
\hline Name \\
\hline Company (if applicable) \\
\hline Address (Street plus Apt or Ste) \\
\hline City & \\
\hline Country & Postal Code (9-digit ZIP for US) \\
\hline Telephone & Fax \\
\hline E-Mail &
\end{tabular}

NEW Information (Please print.)

\begin{tabular}{lc}
\hline Name \\
\hline Company (if applicable) \\
\hline Address (Street plus Apt or Ste) \\
\hline City & \\
\hline Country & Postal Code (9-digit ZIP for US) \\
\hline Telephone & Fax \\
\hline E-Mail &
\end{tabular}

